\title{
The transcriptional activity of HERV-I LTR is negatively regulated by its cis-elements and wild type p53 tumor suppressor protein
}

\author{
Nien-Tzu Chang ${ }^{1,2}$, Wen K. Yang ${ }^{2,3, *}$, Huey-Chung Huang ${ }^{4}$, Kai-Wun $\mathrm{Yeh}^{5} \&$ \\ Cheng-Wen $\mathrm{Wu}^{2,6, *}$ \\ ${ }^{1}$ Graduate Institute of Life Science, National Defense Medical Center, Taipei, Taiwan; ${ }^{2}$ Institute of \\ Biomedical Sciences, Academia Sinica, Taipei, Taiwan; ${ }^{3}$ China Medical University Hospital, 2 Yu-ter Road, \\ Taichung, 404, Taiwan; ${ }^{4}$ Department of Biochemistry, National Taiwan University, Taipei, Taiwan; \\ ${ }^{5}$ Department of Botany, National Taiwan University, Taipei, Taiwan; ${ }^{6}$ National Health Research Institutes, \\ Miaoli County, Taiwan, ROC
}

Received 14 August 2006; accepted 24 September 2006

(c) 2006 National Science Council, Taipei

Key words: human endogenous retrovirus I type, long terminal repeat, negative regulatory element, wild type p53, mutant p53

\begin{abstract}
Human endogenous retroviruses (HERVs), abundantly inter-dispersed in the genome, carry long terminal repeats (LTRs) that may potentially retro-transpose to new genomic sites and deregulate the neighboring cellular genes. However, normally HERVs are either structurally defective or inactive due possibly to stringent negative control mechanisms. To study the possible negative regulation of HERV, we isolated the LTR of RTVL-Ia and constructed site-specific mutations for analysis of the promoter and enhancer functions by using chloramphenicol acetyl transferase (CAT) reporter assay. Our results showed that in most transfected human cells the LTR-mediated CAT expression was negligible unless a sequence segment at the AGTAAA polyadenylation site was deleted. In addition, we have found that the wild type p53 may inhibit whereas a p53 mutant (V143A) stimulate the transcriptional activity of HERV-I LTR. Our results imply that HERV-I LTR, while under negative control by its LTR cis-elements and by wild type p53, may become active upon p53 mutation.
\end{abstract}

\section{Introduction}

Human genome, like those of other organisms, harbor middle-repetitive gene families of interdispersed provirus-like DNA sequences that are presumably generated by retrovirus infection and germline integration during phylogenetic evolution [1-3]. A full-length HERV gene generally contains sequences similar to proviral DNA of gag, pol, env structure genes bounded by the insertional long

*To whom correspondence should be addressed. (W.K. Yang); Tel: +886-4-2205-3366; Fax: +886-4-2207-9649; E-mail: wkyang@mail.cmu.edu.tw; (C-W.Wu), E-mail: ken01@nhri. org.tw terminal repeats (LTRs) of several hundred nucleotides, in which the promoter and enhancer function are located $[4,5]$. It is known that infectious retroviruses are potentially pathogenic and carcinogenic by de novo insertion of proviral DNA, leading to LTR-driven activation of neighboring proto-oncogenes or disruption of important cellular genes including tumor suppressor genes $[6,7]$. Reports of the detection of HERV mRNA, HERV proteins and even HERV particles in human pathological conditions, especially in cancer cells, are increasing in recent years [8-10]. However, in comparison to endogenous retroviruses of other vertebrates, notably chicken and mice [11, 12], HERVs are expressed much rarely in 
the normal human somatic cells and hardly transpose in the germline during recent human evolution [13]. In this respect, very little is known about the transcriptional regulation of HERV, the first step of retro-transposition process, with respect to the LTR cis-elements interacting with tissue-specific and/or pathogenesis-associated nuclear transacting factors, as found in a previous study on chromosomal endogenous retroviruses of the mouse [14].

RTVL-Ia, a representative member of the HERV type I (HERV-I) family with a characteristic isoleucine tRNA primer binding site was originally discovered within the intron of haptoglobin-related (HPR) locus on chromosome 16 [15]. According to phylogenetic analysis [13], HERV-I genes are among the most anciently integrated HERVs in the human genome. Very little is known about the RNA and protein expression of HERV-I except recent microarray data showing its presence in the testis and uterus tissues [16], The biological significance of this selective expression remains to be elucidated. Since HERV-I gene expression is much less than HERV$\mathrm{H}$, HERV-K and HERV-W in most human cell lines examined (our unpublished data), the transcriptional activity of HERV-I LTR might be very weak or repressed by negative regulation.

To define the regulatory cis-elements of HERV-I LTR, we have molecularly cloned the RTVL-Ia LTR and constructed series of recombinant plasmids carrying chloramphenicol acetyl transferase (CAT) gene as the reporter and various sitespecific deletion and site-directed mutations on CAAT element, TATA element, and polyadenylation element of HERV-I LTR. Using transient DNA transfection and CAT assay for functional activities of these LTR-CAT constructs, we have located a negative regulatory function to the AGTAAA polyadenylation site of HERV-I LTR. We also observed that the expression of the transfected LTR-CAT constructs varied considerably in human cervical cancer cells apparently according to the tumor suppressor p53 status, i.e. low in wild type p53-harboring $\mathrm{HeLa}, \mathrm{SiHa}$ and CC-7T cells but high in C33A and HT-3 cells with a mutated $\mathrm{p} 53$. To determine the effects of $\mathrm{p} 53$ on the transcriptional activity of HERV-I LTR, p53null Saos-2 cells were co-transfected with the LTRCAT constructs and an expression plasmid of wild-type 53 (wtp53) or a mutant p53 with an altered amino acid at position 143, 175, 248, 273 or 281. Our results showed that, while one p53 mutant (V143A) activated the promoter activity significantly, wtp53 efficiently down-regulated the promoter activity of HERV-I LTR by acting on both the TATA and CAAT elements.

\section{Materials and methods}

\section{Cell lines}

CC-7T, a Chinese-derived cervical cancer cell line, was a gift from Dr. C.-M. Chang of Tri-Service Veterans General Hospital (Taipei, Taiwan, R.O.C.). Other human cervical carcinoma cell lines, HeLa, SiHa, C33A and HT-3 were purchased from the American Type Culture Collection (ATCC, Rockville, MD, USA). These cells were grown in Dulbecco modified Eagle medium (DMEM) supplemented with $10 \%$ fetal calf serum (FCS). Human osteogenic sarcoma cell, Saos-2, was purchased from the American Type Culture Collection (ATCC, Rockville, MD, USA) and cultured in McCoy's 5A medium supplemented with $15 \%$ FCS. All cells were incubated at $37{ }^{\circ} \mathrm{C}$ in $100 \%$ humidity and a $5 \% \quad \mathrm{CO}_{2}$ atmosphere.

\section{Plasmids construction}

Four pCAT reporter vectors, pCAT-basic (pCATB), pCAT-control (pCAT-C), pCAT-enhancer (pCAT-E) and pCAT-promoter (pCAT-P), were obtained from Promega (Madison, WI, USA) for promoter and enhancer function assay. HERV-I LTR was made by PCR using two 22-mer oligonucleotides (5'-GCCTGCTTAAGTTTCCTTGTCC-3' and 5'-TTTCCCTGTATGGGCCACC AAA- $3^{\prime}$ ) corresponding to the RTVL-Ia sequence. The amplified DNA fragment was subcloned into the pCAT-E (containing SV40 enhancer) and pCAT-P (containing SV40 early promoter) for detecting the promoter and the enhancer activities, respectively. The deletion mutants were constructed by blunt end ligation of each LTRrestricted fragment into the SalI site of the pCAT-E or pCAT-P and the point mutation mutants were obtained by site-directed mutagenesis of IE123 or IP123 using Transformer ${ }^{\mathrm{TM}}$ sitedirected mutagenesis (Clontech Laboratories, Palo 
Alto, CA, USA). All these plasmid constructs were further confirmed by DNA sequence determination.

pCEPwtp53 plasmid expressing wild type p53 was constructed by inserting a PCR fragment containing the whole p53 coding sequence with HindIII/BamHI sites into pCEP4 (Invitrogen). pCEP-p53 mutants carrying the p53 gene with various mutation at codon 143 ( $\mathrm{V}$ to $\mathrm{A}$ ), 175 ( $\mathrm{R}$ to H), 248 (R to W), 273 (R to H) or 281 (D to $G$ ) were constructed by point-mutation of pCEPwtp53 using QuikChange II XL site-directed mutagenesis kit (Strategene). All the p53 constructs, named pCEPwtp53 (containing wtp53 gene), mt1(V143A) (containing mutant p53 with $\mathrm{V}$ to $\mathrm{A}$ at codon 143), $\mathrm{mt} 2(\mathrm{R} 175 \mathrm{H}), \mathrm{mt} 3(\mathrm{R} 248 \mathrm{~W})$, $\mathrm{mt} 4(\mathrm{R} 273 \mathrm{H})$ and $\mathrm{mt5}(\mathrm{D} 281 \mathrm{G})$, were confirmed by DNA sequencing.

\section{DNA transfection}

Transient transfection of plasmid DNAs was performed mainly according to the calcium phosphate method described by Sambrook [17]. Briefly, cells were seeded at $5 \times 10^{5}$ cells $/ 60-\mathrm{mm}$ dish 18 $24 \mathrm{~h}$ before transfection and medium was refreshed $4 \mathrm{~h}$ before adding calcium phosphateDNA precipitates. Ten microgram of plasmid per $60 \mathrm{~mm}$ dish was used for each transfection. The cells were exposed to the calcium phosphate-DNA precipitates for $16 \mathrm{~h}$, and then shocked with $15 \%$ glycerol solution for $3 \mathrm{~min}$ at $37^{\circ} \mathrm{C}$. Cells were harvested for CAT assay three days after DNA transfection.

\section{CAT assays}

Cells were harvested, suspended in $100 \mu \mathrm{l}$ of $0.25 \mathrm{M}$ Tris buffer ( $\mathrm{pH} 7.8$ ), lysed by three cycles of freezing and thawing. The cell lysates was centrifuged at $12,000 \times g$ for $20 \mathrm{~min}$ at $4{ }^{\circ} \mathrm{C}$ in an Eppendorf microfuge. The protein concentration of the supernatant was determined by BCA protein assay (Pierce Corp., Rockford, IL, USA) and the CAT activity of the cell lysates was determined with $\left[{ }^{14} \mathrm{C}\right]$ chloramphenicol (Amersham, Buckinghamshire, UK) as a substrate. An equal amount of protein was mixed with $0.15 \mu \mathrm{Ci}$ of $\left[{ }^{14} \mathrm{C}\right]$ chloramphenicol, $3 \mathrm{mM}$ acetyl-coenzyme A (Sigma, St Louis, MO, USA), and 0.25 M Tris buffer ( $\mathrm{pH}$ 7.8), and the reaction mixtures were incubated at $37{ }^{\circ} \mathrm{C}$ for one hour and extracted with ethyl acetate, dried under vacuum and dissolved in $20 \mu \mathrm{l}$ of ethyl acetate. The final reaction mixtures are analyzed by thin-layer chromatography on silica gel plates (Merck, Darmstadt, Germany) in a solution of $95 \%$ chloroform$5 \%$ methanol. The radioactive products were visualized by autoradiography, and the CAT activity was quantified by densitometry of the film based on the ratio of $\left[{ }^{14} \mathrm{C}\right]$ chloramphenicol converted to the acetylated form. For comparison of different LTR-CAT constructs, CAT activities were expressed relative to the activity of positive control pCAT-C, which were rather dependable under the set conditions of an experiment, although the ratio may vary in different experiements due to variations in transfection efficiencies and CAT assay conditions.

\section{Results}

Functional analysis of the deletion mutants of HERV-I LTR

The elements of CCAAT, ATAAAAA and AGTAAA, designated as box 1, box 2 and box 3 , in the DNA sequence of the HERV-I LTR clone (Figure 1) are consistent with the previously reported transcriptional elements of retroviral genes. To define the individual as well as the combinatorial functions of these three cis ele-

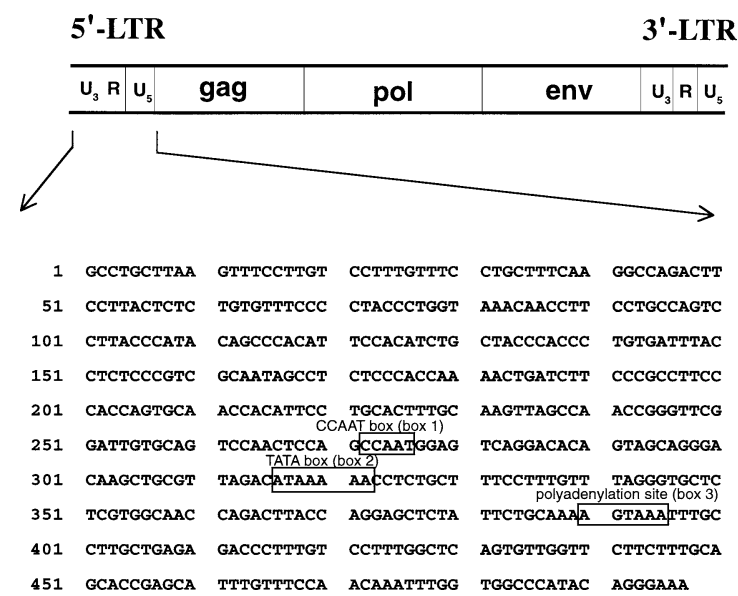

Figure 1. Complete nucleotide sequence of a HERV-I 5'-LTR is shown with the consensus CCAAT (Box 1), TATA (Box 2) and poly-A (Box 3) elements indicated in boxes. 


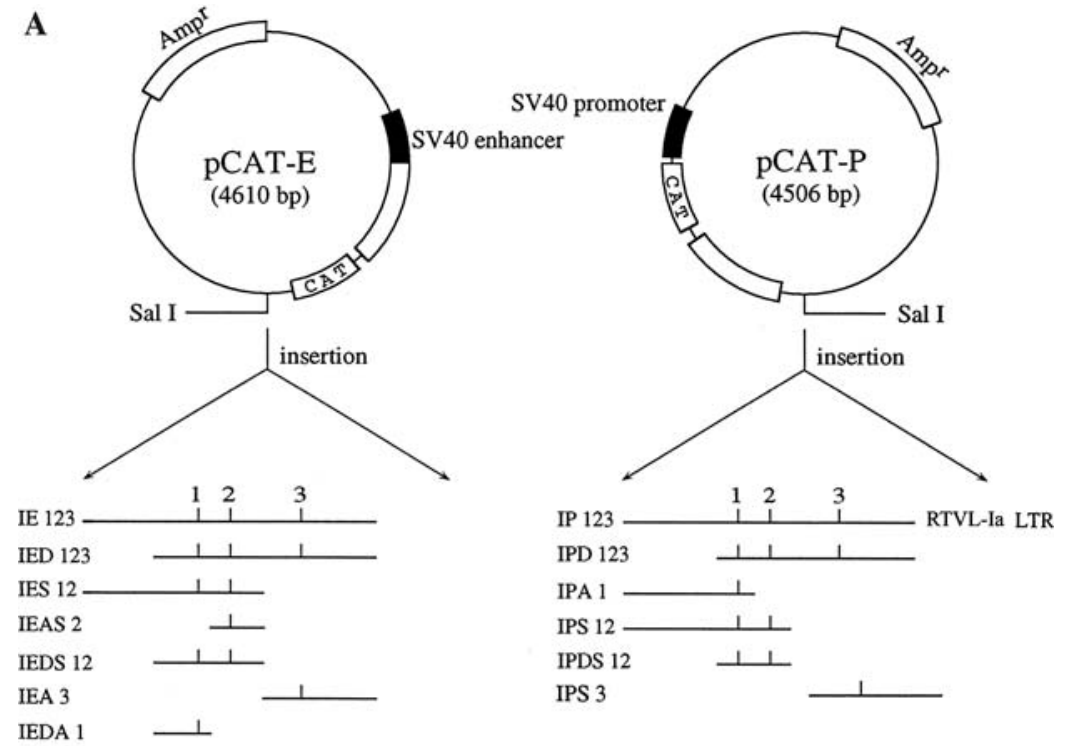

B Assay for the promoter function
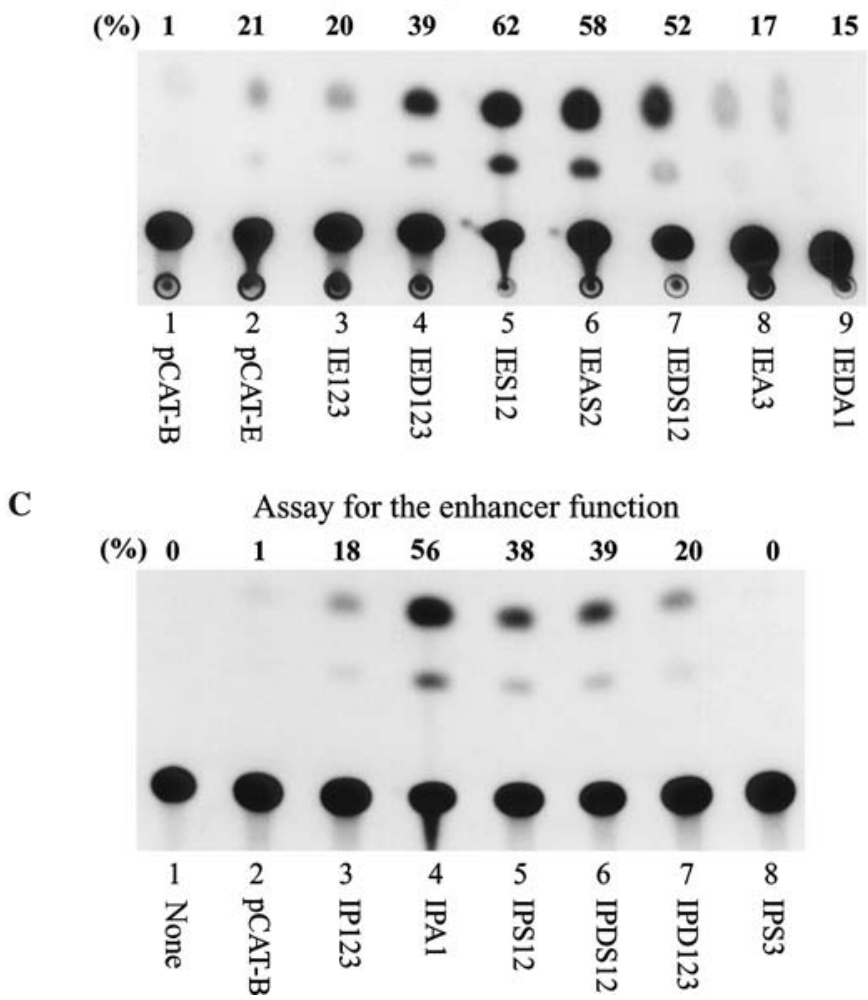

Figure 2. The promoter and the enhancer activities of HERV-I 5'-LTR determined by fragment deletion. (A) Two series of the promoter reporter and the enhancer reporter recombinant plasmids were constructed by insertion of LTR or a LTR sub-fragment (with certain fragments deleted) into pCAT-E and pCAT-P at the SalI site of the polycloning region. (B) and (C) The LTR deletion constructs were transfected into CC-7T cells and assayed for the promoter and enhancer activity. Autoradiograms of thinlayer chromatography show the CAT activities of different pCAT-E and pCAT-P LTR deletion constructs. Upper panel of the autoradiograph shows percentage of chloramphenicol acetylation and the lower panel shows the name of plasmid clones. The pCAT-P was not shown on the plate, its chloramphenicol acetylation percentage was 21 in the same experiment. The result is reproducible in three independent experiments. 
ments, we dissected the LTR sequence into various fragments, each containing one to three elements. The whole HERV-I LTR and the dissected LTR fragments were each inserted into the SV40 enhancer-containing pCAT-E upstream of the CAT gene for promoter assay, and also each inserted into the SV40 promoter-containing pCAT-P for enhancer assay (Figure 2A). The plasmid pCAT-B that contains neither promoter nor enhancer was used as the negative control while pCAT-C that contains both the SV40 early promoter and SV40 enhancer served as the positive control in the functional assays. Initial experiments by transfection into human cervical cancer CC-7T cell line showed that the CAT expression from the whole LTR-containing IE123 and IP123 was not different, or even lower than, from the parental pCAT-E and pCAT-P plasmids (Figure 2 and unshown data) and that only from constructs of dissected LTR with certain fragmentdeletion could we detect significant increases of CAT expression over the parental plasmids (Figures $2 \mathrm{~B}$ and $2 \mathrm{C}$ ). Similar results were obtained by using other human cancer cells, including HeLa, SiHa, H1A and CG1 lines, as the transfection recipient (data not shown), suggesting a poor transcriptional activity of this HERV-I LTR as a whole and also some negative effects of HERV-I

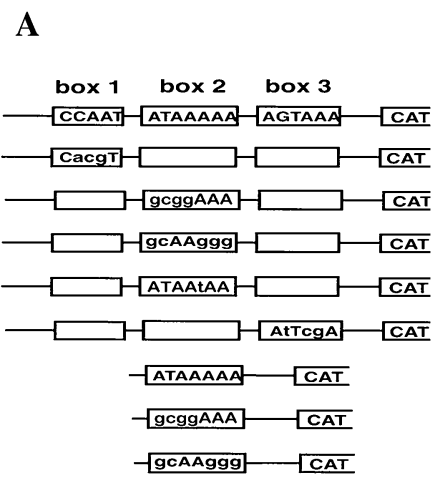

$\mathbf{B}$

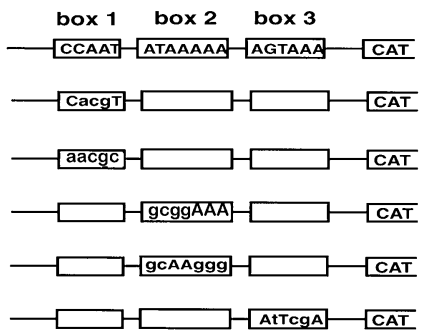

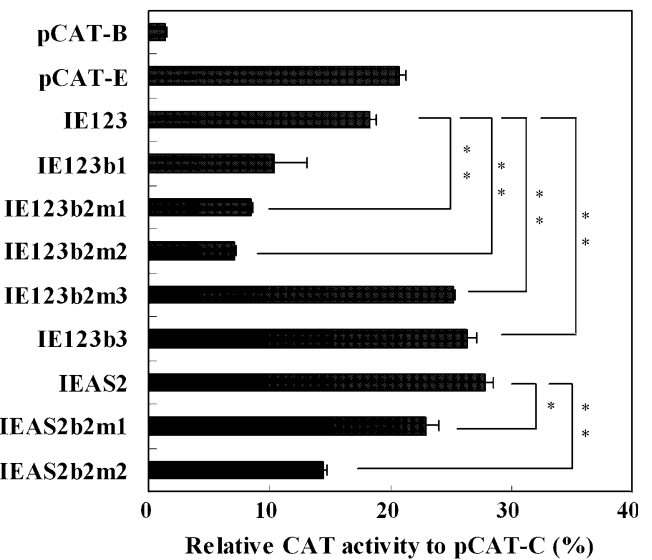

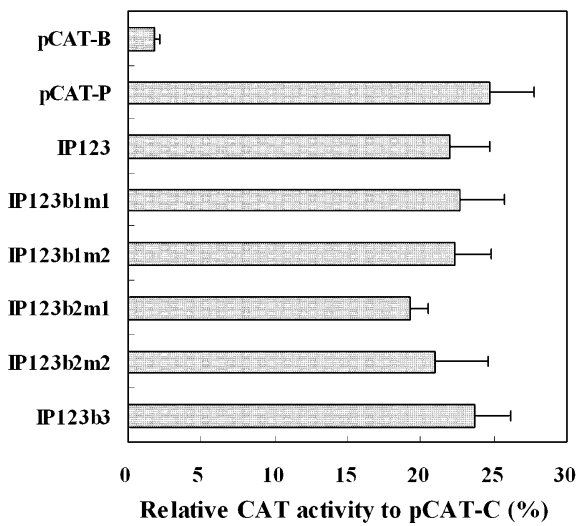

Figure 3. Determination of transcriptional activities of HERV-I 5'-LTR constructs carrying various point mutations. (A) The left schematic diagram shows the nucleotides change by site-directed mutation of box 1, box 2 and box 3 in IE123 and IEAS2, named IE123b1, IE123b2m1, IE123b2m2, IE123b2m3, IE123b3, IEAS2b2m1 and IEAS2b2m2. The site-directed mutation mutants were transfected into CC-7T cells and assayed for promoter activity. The right panel graph shows the relative promoter activity of mutation constructs as the percentage of the CAT activity of pCAT-C. (B) The left schematic diagram shows the nucleotide changes by site-directed mutagenesis of box 1, box 2 and box 3 in IP123, named IP123b1m1, IP123b1m2, IP123b2m1, IP123b2m2 and IP123b3. The right panel graph shows the relative enhancer activity of mutation constructs as a percentage of the CAT activity of pCAT-C. The average and standard deviations are from three independent experiments, $* p<0.05$ and $* * p<0.01$ versus IE123 or IEAS2 respectively. 


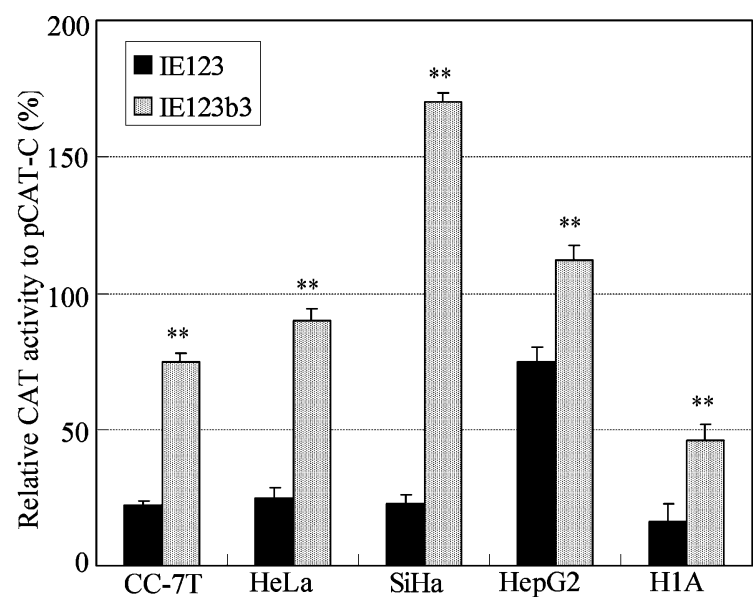

Figure 4. Promoter activities of HERV-I LTR and its sitedirected mutant, IE123b3, in different cell lines. The relative promoter activity of IE123 and IE123b3 is given as a percentage of the CAT activity of pCAT-C in the respective recipient cells. The average and standard deviations are calculated from data of two independent experiments, $* p<0.05$ and $* *$ $p<0.01$ versus IE123.

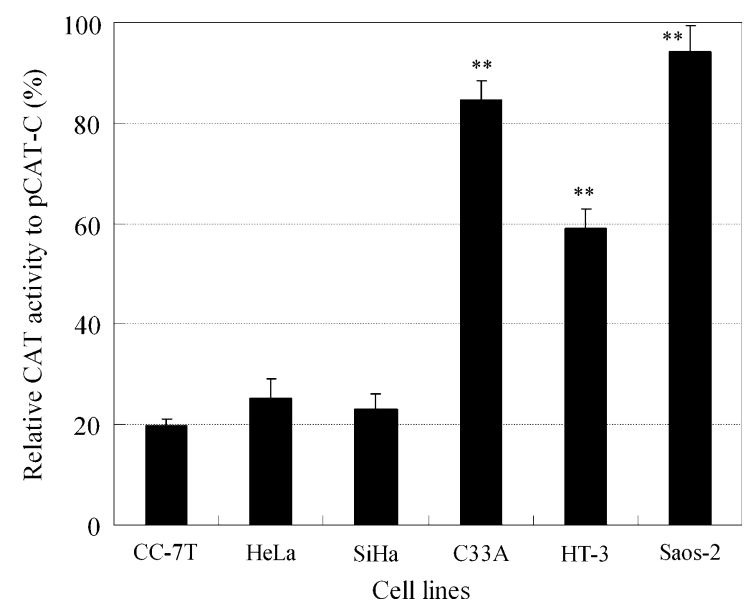

Figure 5. Promoter activities of HERV-I LTR in various human cancer cell lines with different p53 status. Plasmid of IE123 was transfected into the HPV-positive cervical cancer HeLa, SiHa and CC-7T cells, the HPV-negative cervical cancer C33A and HT-3 cells, and p53-null human osteosarcoma Saos-2 cells. The relative promoter activity of IE123 was shown as the percentage of the CAT activity of pCAT-C in the respectively transfected cells. The average and standard deviations are calculated from three independent experiments, $* p<0.05$ and $* * p<0.01$ versus CC-7T.

LTR on the SV40 enhancer and promoter. For example, using CC-7T cells for the promoter function assay and the parental pCAT-E for comparison, we found strong promoter activity with IES12 and IEDS12 that contained box 1 and box 2 without box 3 whereas very little promoter activity with IEDA1 and IEA3 that contained, respectively, only box 1 and only box 3 (Figure 2B). In particular, IEAS2 that contained only box 2 showed the strongest promoter activity among the LTR-CAT recombinant constructs. These results indicate that LTR fragments that carry the ATAAAAA element may confer a promoter function. In enhancer function assay (Figure 2C), IPS12 and IPDS12 that contained box 1 and box 2 without box 3 possessed strong enhancer activity and IPA1 that contained box 1 alone gave particularly the strongest enhancer activity, whereas IPS that contained only box 3 showed no enhancer activity, suggesting the association of the CCAAT element with the enhancer function of this HERV-I LTR.

\section{Functional analysis of site-directed mutants of HERV-I LTR}

To further define the promoter and enhancer functions of the specific elements, we prepared a series of point mutations at box 1 or box 2 or box 3 in IE123 and IP123. The schematic diagram of the site-directed mutagenesis is shown in Figure 3. In the promoter function assay of site-directed mutation constructs (Figure 3A), changes from the wild type sequence ATAAAAA in IE123 or IEAS2 to GCGGAAA (in IE123b2m1 or IEAS $2 \mathrm{~b} 2 \mathrm{~m} 1$ ) and to GCAAGGG (in IE $123 \mathrm{~b} 2 \mathrm{~m} 2$ or IEAS2b2m2) resulted in a decrease in CAT expression. Also, mutation of the wild type sequence CCAAT (in IE123) into CACGT (in IE123b1) decreased the promoter activity. In contrast, change from the wild-type ATAAAAA sequence into a typical TATA box sequence, ATAATAA, (from IE123 to IE123b2m3) markedly increased the CAT activity. The result suggested that ATAAAAA element exerts mainly a promoter function.

In the enhancer activity assays (Figure 3B), IP123b1m1, IP123b1m2, IP123b2m1 and IP123 b2m2 did not cause a significant difference in enhancer activity, as compared to wild type IP123. Since all the site-directed IP mutants showed lower CAT expression than pCAT-P, we concluded that overall HERV-I LTR had poor enhancer activity even with the CCAAT element to provide a partial enhancer function. 
AGTAAA element plays a negative regulatory role in the HERV-I LTR promoter and enhancer activity

AGTAAA, which is present in the mouse mammary tumor virus (MMTV) LTR as the polyadenylation signal [18], is found in both the $5^{\prime}$ - and $3^{\prime}$ LTRs of HERV-I. In the promoter function assay of LTR fragments (Figure 2B), IES12 and IEDS12 each expressed higher CAT level than IE123. Similarly, in the enhancer function assay (Figure 2C), IPS12 and IPD123 each gave higher CAT activities than IP123. The results showed that promoter and enhancer activities could be markedly increased by deleting box 3 from full-length LTR, implicating a negative regulatory function within box 3 . We therefore performed site-specific mutagenesis of the box 3 in the IE123 and IP123 constructs to refine this negative regulatory element. The derivative of IE123 with AGTAAA element mutated to ATTCGA, called IE123b3, was found to express higher CAT activity than the original IE123 (Figure 3A). And the site-directed mutant of IE123b3 showed higher CAT activities than IE123 in HeLa, SiHa, HepG2 and H1A cells similarly as in CC-7T cells (Figure 4). Taken together, these results demonstrated that the AGTAAA element of the putative polyadenylation signal, at least in part, exerted a negative regulatory effect on the transcriptional activity of HERV-I 5'-LTR.

Both the promoter and enhancer activities of HERV-I LTR are low in HPV-positive cervical carcinoma cells but high in HPV-negative cervical carcinoma cells

In this study, we have used five cervical carcinoma cell lines, including HPV-positive HeLa, SiHa and CC-7T, and HPV-negative C33A and HT-3, and one osteosarcoma Saos-2 cell line as recipients of DNA transfection for determining the transcriptional activity of HERV-I LTR. The HPV positive cells expressed low levels of wtp53 protein as a consequence of its association with the HPV E6 onco-proteins, and the HPV negative cells have mutations in the p53 gene and expressed mutated p53 protein $[19,20]$. We noted that CAT activities of IE123 were consistently much lower in the wild type p53-harboring $\mathrm{HeLa}, \mathrm{SiHa}$ and $\mathrm{CC}-7 \mathrm{~T}$ cells than in mutant p53-harboring C33A, HT3 and p53-null Saos-2 cells (Figure 5). Similar results were obtained using IP123 (data not shown). The results implied that the transcriptional activity of LTR might be affected by the p53 phenotypes in these cancer cells.

Transient expression of wild type p53 represses while mutant p53 stimulates the transcriptional activity of HERV-I LTR

To confirm that the tumor suppressor p53 plays a role in regulating transcriptional activity of HERV-I LTR, we examined the transcriptional activity of LTR-CAT constructs in Saos-2 cells with exogenously transfected p53. The plasmid pCEPwtp53 or one p53 mutant (V143A) was cotransfected respectively with plasmid IE123 or IP123 into p53-null Saos-2 cells to examine the effect on CAT expression (Figure 6A). The CAT activities of IE123 and IP123 were reduced in cells cotransfected with pCEPwtp53 but increased in cells cotransfected with p53 mutant of V143A. Similar results were obtained by using C33A cells (data not shown). Other p53 mutants, specifically mt1(V143A), mt2(R175H), mt3(R248W), $\mathrm{mt} 4(\mathrm{R} 273 \mathrm{H})$ and $\mathrm{mt5}(\mathrm{D} 281 \mathrm{G})$ were cotransfected individually with plasmid IE123 into Saos-2 cells and assayed for their CAT activities (Figure 6B). The CAT activity of IE123 was reduced in cells cotransfected with pCEPwtp53 but increased in cells cotransfected with one p53 mutant of V143A, as compared to cells cotransfected with pCEP4 vector alone. The results indicated that wtp53 repressed but mutant p53 (V143A) stimulated the promoter activity and enhancer activity of HERV-I LTR.

\section{Wtp53 repression of the HERV-I LTR} transcriptional activity may be mediated through the TATA- and CAAT-like elements

To map the region of LTR that is responsible for the inhibitory effect of wtp53, LTR deletion mutants, IED123, IEDA1, IEAS2 or IEA3, together with pCEPwtp53 were co-transfected into Saos- 2 cells. The CAT activities of the four LTR deletion mutant constructs were inhibited by pCEPwtp53 (Figure 7A). Besides, three LTR point mutants mutation respectively of box 1,2 and 3 (i.e., IE123b1, IE123b2m2 and IE123b3) were co-transfected with pCEPwtp53 into Saos-2 cells. The CAT activities of these mutant con- 


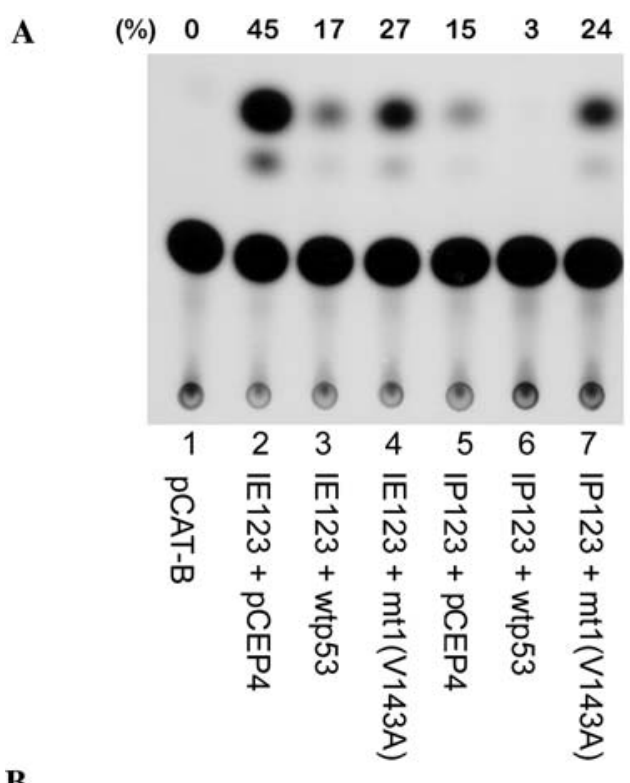

(\%)

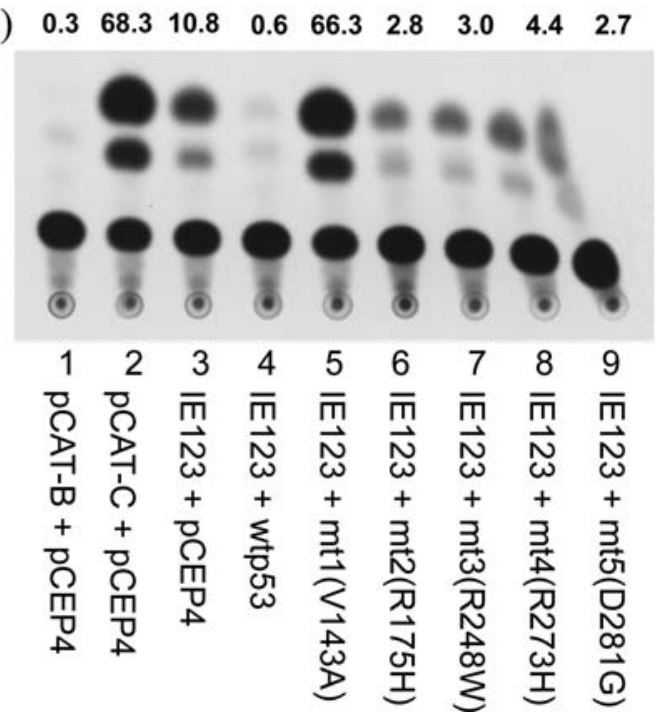

Figure 6. Effects of exogenously transfected wtp53 or mutant p53 on the transcriptional activity of HERV-I LTR. (A) IE123 or IP123 were mixed with wtp53 (expressing wild type p53) or mt1(V143A) and co-transfected into Saos-2 cells and the CAT activity of the transfectants was determined by thinlay chromatography and autoradiography. (B) IE123 was cotransfected with either pCEP4 (vector only), or wtp53, or one of the p53 mutants, mt1(V143A), mt2 (R175H), mt3 (R248), $\mathrm{mt} 4(\mathrm{R} 273 \mathrm{H})$ and $\mathrm{mt} 5(\mathrm{D} 281 \mathrm{G})$ into Saos-2 cells. The CAT activity of the transfectants was determined by thin-layer chromatography and autoradiography. The upper panel of autoradiograph shows the percentage of chloramphenicol acetylation quantitation and the lower panel shows the name of plasmid clones. The result is reproducible in three independent experiments. structs were also repressed by wtp53 (Figure 7B), implying that wtp53 exerted its effect mainly on the box 1 CCAAT element and the box 2 ATAAAAA element in the HERV-I LTR promoter region. Taken together, the results of LTR fragment and point mutation constructs revealed that wtp53 inhibits HERV-I LTR promoter activity mainly via the regions of TATA- and CAATelements.

\section{Discussion}

We have engaged in a series of studies to understand the control mechanisms of multiple endogenous retroviral genes in the human genome. The focus of present investigation is to understand the LTR-directed gene regulatory mechanism of HERV-I in the context of ciselements in naked (non-methylated non-histone associated) DNA form. For this purpose, we constructed series of CAT recombination plasmids with molecularly cloned RTVL-Ia LTR and its site-specific deletion and mutation derivatives for functional assay in various human cancer cells. We have found that, unlike the active LTRs of the HERV-H [21], HERV-K [22] and HERV-W [23] in vitro, the full length HERV-I LTR has very poor promoter and enhancer activity. However, we have found that deletion of the box-3 LTR fragment that contains AGTAAA element or site-directed mutation at this site can increase the transcriptional activity of HERV-I LTR, suggesting the presence of a negative regulatory role played by this putative polyadenylation AGTAAA signal. This is a distinct controlling mechanism not found in other HERV LTRs examined. More significantly, we have found that the wild type p53 can downregulate the transcriptional activity of HERV-I LTR through its action on the promoter and enhancer sites, while p53 mutant of V143A produced the opposite function of up-regulation. These results suggest that the LTR-directed expression of HERVs may be altered in p53mutanted human cancer cells.

In contrast to the proviruses of exogenous retroviral infection that tends to continue their 

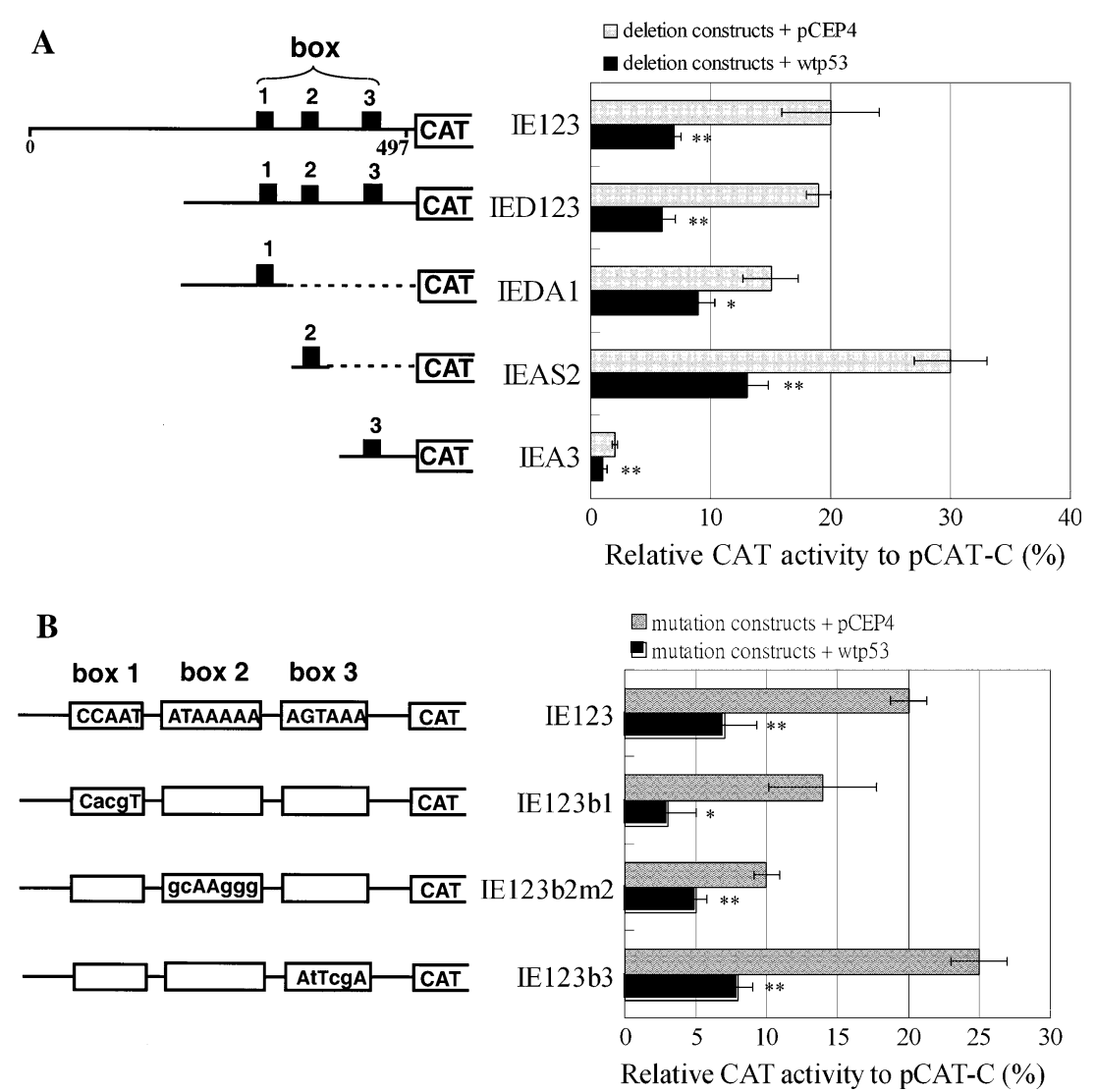

Figure 7. Effect of wtp53 on the promoter activities of various deletion and site-directed mutation mutants of LTR-CAT. (A) The LTR deletion constructs with box 1, box 2, or box 3 are indicated on the left, and relative CAT activities of these deletion constructs co-transfected with/without wtp53 in Saos-2 cells are shown on the right. (B) The LTR site-directed mutation constructs carrying point mutations in box 1, box 2 or box 3 of IE123 plasmid, designated IE123b1, IE123b2m 2 and IE123b3 are indicated on the left, and relative CAT activities of the point-mutation constructs co-transfected with/without wtp53 in Saos-2 cells are shown on the right. The relative promoter activity is shown as the percentage of the CAT activity of pCAT-C. The average and standard deviations are from three independent experiments, ${ }^{*} p<0.05$ and $* * p<0.01$ vs pCEP4 construct.

replication cycle and horizontal transmission, most HERVs in the human genome are defective in the viral structure genes and can not by themselves generate infectious retroviruses. In addition, HERVs are relatively inactive presumably due to strict negative control mechanisms of their transcription through the cis elements in the LTR. Indeed, negative regulatory elements (NREs) have been identified in HERV-K LTR [22], HERV-H LTR [24] and other endogenous retroviral LTRs. The use of putative polyadenylation site, AGTAAA, as a NRE in the HERV-I LTR might represent a distinct mechanism. It is however not known whether or not the AGTAAA element, being located downstream of the promoter and transcription initiation sites in the $5^{\prime}$
LTR as in the $3^{\prime}$ LTR, would hinder the progression of RNA polymerase initiation complexes, thus preventing transcription or causing premature termination. Furthermore, we have found ATAAAAA sequence at the TATA box of our HERV-I LTR clone instead of the typical TATA box found in the LTRs of HERV-H, HERV-K and HERV-W gene families. And site-specific mutation of the TATA box from ATAAAAA to ATAATAA significantly increased the promoter activity (Figure 3A, IE123 vs. IE123b2m3). Since both HERV-I and HERV-E that carry the same atypical TATA are among the low expressing HERV families, the transcriptional activity of HERV LTR might have been attenuated by mutation(s) at the promoter site during phyloge- 
netic evolution. Taken together, the NRE of AGTAAA in combination with an atypical TATA box plays a negative role in control of the transcriptional activity of HERV-I LTR.

Although the RNA transcripts of HERV-I could hardly be detected in most human somatic cells by conventional methods [25], recent microarray analysis showed tissue-specific expression of HERV-I in reproductive organs [16]. It implied that HERV-I gene expression was not only regulated by its cis-elements but also by tissue-specific factors. In our functional assay of IE123 and IP123 in different cervical cancer cell lines, we found the CAT activity of both IE123 and IP123 was low in the HPV-positive cell lines, CC7T, SiHa and HeLa, but ten fold-higher in HPV-negative cervical cancer cell lines, C33A (with p53 mutation at amino acid position 273) and HT-3 (with p53 mutation at amino acid position 245) [26] We next determined whether the status of p53 would regulate the transcriptional activity of HERV-I LTR, and found that the CAT activity of IE123 was strongly repressed by wtp53 and, in contrast, markedly stimulated by a mutant $\mathrm{p} 53$ with mutation at codon 143. The results suggest that $\mathrm{p} 53$ can modulate the transcriptional activity of HERV-I LTR. According to previous reports, wtp53 is potentially an inhibitor, whereas mtp53 an activator, of several viral promoters, including the herpes simplex virus type I UL9 promoter, the human cytomegalovirus major immediate early promoter enhancer, and the LTR promoters of Rous sarcoma virus and human T-cell lymphotropic virus type I [27, 28] It is likely that HERV-I LTR, like other exogenous retrovirus promoters, is repressed by wtp53 and activated by mtp53, especially by mutant p53 (V143A). Besides, wtp53 was reported to exert more generalized repression of transcription on various genes by the direct interaction with transcriptional factors, such as TATA- binding protein (TBP) [29-31], Sp1 [32], and CCAAT-binding factors [33] In combination with our observations that the loss of expression of HERV-I results from the absence of transcriptional activation through both the TATA and CAAT sites, we presume that the repression of HERV-I LTR by wtp53 may be mediated via the specific interaction of p53 with TBP and p53 with the CAAT-binding factor. In addition, it appears that mtp53 did not behave merely as losing the function of wtp53 in our LTR functional analysis. Several studies revealed that certain types of $\mathrm{p} 53$ mutations will result in gain of functions and exert functions that the wild type p53 does not [34] For example, human p53 mutant alleles (mutation at codon 143, $175,248,273$ and 281) have been shown to either enhance the tumorigenic potential in nude mice or increase the plating efficiency in soft-agar cell culture [35]. Similarly, the mutant p53 (V143A) might confer the gain-of-functions by activating the promoter activity of HERV-I LTR. In this respect, as HERV LTRs exist abundantly in the human genome, it would be of interests to determine if p53 mutation might enlist HERVs into the carcinogenesis process by LTR activation to produce retro-transposition factors and also to cause aberrant regulation of upstream- and downstreamproto-oncogenes and tumor suppressor genes.

In this study we have elucidated some control mechanisms of HERV, particularly the identification of negative cis-elements and the finding of regulatory modulation by $\mathrm{p} 53$ proteins, by using the HERV-I LTR model at the level of unmodified LTR DNA sequences. However, the transcriptional regulation of the abundant interdispersed HERVs in human genome may also occur at the level of chromatin structure, which is known to be modified by DNA methylation and histone acetylation. We have therefore investigated the expression of indigenous HERVs in human cells transfected with the wild-type and mutant p53s, with or without exposure to inhibitors of DNA methylation and histone acetylation, and obtained consistent and additional results that the expression of chromosomal HERV family genes, especially with inhibited DNA methylation and histone acetylation, is stimulated by some p53 mutants in human cells in vitro (manuscript in preparation). Furthermore, it remains to be investigated whether or not regulation of HERV expression can take place at the level of tissuespecific transcriptional and LTR binding nuclear factors that may show dynamic changes in response to environmental hazardous conditions like carcinogen exposure, as previously reported for the mouse endogenous retroviral LTR sequences [14].

\section{Acknowledgments}

We dedicate this paper to the late Dr. Felicia Y.-H. Wu, a brave fighter of cancer, while serv- 
ing as a research fellow at Institute of Biomedical Sciences, Academia Sinica, Taipei, Taiwan and participating in this HERV study till the end of her life. This study was funded in part by a R.O.C. National Science Council grant (WKY).

\section{References}

1. Leib-Mosch C., Brack-Werner R., Werner T., Bachmann M., Faff O., Erfle V. and Hehlmann R., Endogenous retroviral elements in human DNA. Cancer Res. 50: 5636S5642S, 1990.

2. Cohen M. and Larsson E., Human endogenous retroviruses. Bioessays 9: 191-196, 1988.

3. Wilkinson D.A., Mager D. and Leong J.A.C., Endogenous human retroviruese. Plenum Press, NY, 1994, pp. 465-535.

4. O'Connell C.D. and Cohen M., The long terminal repeat sequences of a novel human endogenous retrovirus. Science 226: 1204-1206, 1984.

5. Goodchild N.L., Freeman J.D. and Mager D.L., Spliced HERV-H endogenous retroviral sequences in human genomic DNA: evidence for amplification via retrotransposition. Virology 206: 164-173, 1995.

6. Temin H.M., Function of the retrovirus long terminal repeat. Cell 28: 3-5, 1982.

7. Kung H.J., Boerkoel C. and Carter T.H., Retroviral mutagenesis of cellular oncogenes: a review with insights into the mechanisms of insertional activation. Curr. Top. Microbiol. Immunol. 171: 1-25, 1991.

8. Karlsson H., Bachmann S., Schroder J., McArthur J., Torrey E.F. and Yolken R.H., Retroviral RNA identified in the cerebrospinal fluids and brains of individuals with schizophrenia. Proc. Natl. Acad. Sci. USA 98: 4634-4639, 2001.

9. Kempf W., Kadin M.E., Dvorak A.M., Lord C.C., Burg G., Letvin N.L. and Koralnik I.J., Endogenous retroviral elements, but not exogenous retroviruses, are detected in CD30-positive lymphoproliferative disorders of the skin. Carcinogenesis 24: 301-306, 2003.

10. Wang-Johanning F., Frost A.R., Jian B., Epp L., Lu D.W. and Johanning G.L., Quantitation of HERV-K env gene expression and splicing in human breast cancer. Oncogene 22: 1528-1535, 2003.

11. Frankel W.N., Stoye J.P., Taylor B.A. and Coffin J.M., A linkage map of endogenous murine leukemia proviruses. Genetics 124: 221-236, 1990.

12. Yang W.K., Ch'ang L.Y., Koh C.K., Myer F.E. and Yang M.D., Mouse endogenous retroviral long-terminal-repeat (LTR) elements and environmental carcinogenesis. Prog. Nucleic Acid Res. Mol. Biol. 36: 247-266, 1989.

13. Johnson W.E. and Coffin J.M., Constructing primate phylogenies from ancient retrovirus sequences. Proc. Natl. Acad. Sci. USA 96: 10254-10260, 1999.

14. Hoyt P.R., Wang T.H., Henley D.C., Yang D.M., Ch'ang L.Y. and Yang W.K., Carbon tetrachloride induction of rapid changes in liver nuclear protein factors capable of sequence-specific binding to regulatory elements in the long terminal repeat of polytropic-class endogenous murine leukemia virus-related proviruses. Mol. Carcinogen. 8: 245-254, 1993.

15. Maeda N., Nucleotide sequence of the haptoglobin and haptoglobin-related gene pair. The haptoglobin-related gene contains a retrovirus-like element. J. Biol. Chem. 260: 6698-6709, 1985.

16. Seifarth W., Frank O., Zeilfelder U., Spiess B., Greenwood A.D., Hehlmann R. and Leib-Mosch C., Comprehensive analysis of human endogenous retrovirus transcriptional activity in human tissues with a retrovirus-specific microarray. J. Virol. 79: 341-352, 2005.

17. Sambrook J., Fritsch E.F. and Maniatis T., Molecular cloning: a laboratory manual, 2 edn. Cold Spring Harbor Laboratory Press, NY, 1989, pp. 1630-1640.

18. Swain A. and Coffin J.M., Influence of sequences in the long terminal repeat and flanking cell DNA on polyadenylation of retroviral transcripts. J. Virol. 67: 6265-6269, 1993.

19. Scheffner M., Munger K., Byrne J.C. and Howley P.M., The state of the p53 and retinoblastoma genes in human cervical carcinoma cell lines. Proc. Natl. Acad Sci. USA 88: 5523-5527, 1991.

20. von Knebel Doeberitz M., Rittmuller C., Aengeneyndt F., Jansen-Durr P. and Spitkovsky D., Reversible repression of papillomavirus oncogene expression in cervical carcinoma cells: consequences for the phenotype and E6-p53 and E7pRB interactions. J. Virol. 68: 2811-2821, 1994.

21. Feuchter A. and Mager D., Functional heterogeneity of a large family of human LTR-like promoters and enhancers. Nucleic Acids Res. 18: 1261-1270, 1990.

22. Domansky A.N., Kopantzev E.P., Snezhkov E.V., Lebedev Y.B., Leib-Mosch C. and Sverdlov E.D., Solitary HERV-K LTRs possess bi-directional promoter activity and contain a negative regulatory element in the U5 region. FEBS Lett. 472: 191-195, 2000.

23. Lee W.J., Kwun H.J. and Jang K.L., Analysis of transcriptional regulatory sequences in the human endogenous retrovirus W long terminal repeat. J. Gen. Virol. 84: 2229 2235, 2003.

24. Nelson D.T., Goodchild N.L. and Mager D.L., Gain of Sp1 sites and loss of repressor sequences associated with a young, transcriptionally active subset of HERV-H endogenous long terminal repeats. Virology 220: 213-218, 1996.

25. Yeh K.W., Yang W.K., Huang H.C., Feng Y.N., Liu J.C., Wu F.Y. and Wu C.W., Cloning and characterization of the endogenous retroviral-tRNA(Glu) multigene family from human genomes of different racial backgrounds. Gene 155: $247-252,1995$.

26. Liang X.H., Volkmann M., Klein R., Herman B. and Lockett S.J., Co-localization of the tumor-suppressor protein p53 and human papillomavirus E6 protein in human cervical carcinoma cell lines. Oncogene 8: 26452652, 1993.

27. Subler M.A., Martin D.W. and Deb S., Inhibition of viral and cellular promoters by human wild-type $\mathrm{p} 53$. J. Virol. 66: 4757-4762, 1992.

28. Deb S., Jackson C.T., Subler M.A. and Martin D.W., Modulation of cellular and viral promoters by mutant human p53 proteins found in tumor cells. J. Virol. 66: 6164-6170, 1992.

29. Seto E., Usheva A., Zambetti G.P., Momand J., Horikoshi N., Weinmann R., Levine A.J. and Shenk T., Wild-type p53 binds to the TATA-binding protein and represses transcription. Proc. Natl. Acad. Sci. USA 89: 12028-12032, 1992.

30. Liu X., Miller C.W., Koeffler P.H. and Berk A.J., The p53 activation domain binds the TATA box-binding polypeptide in Holo-TFIID, and a neighboring p53 domain inhibits transcription. Mol. Cell Biol. 13: 3291-3300, 1993. 
31. Mack D.H., Vartikar J., Pipas J.M. and Laimins L.A., Specific repression of TATA-mediated but not initiatormediated transcription by wild-type p53. Nature 363: 281283, 1993.

32. Borellini F. and Glazer R.I., Induction of Sp1-p53 DNAbinding heterocomplexes during granulocyte/macrophage colony-stimulating factor-dependent proliferation in human erythroleukemia cell line TF-1. J. Biol. Chem. 268: 7923-7928, 1993.
33. Agoff S.N., Hou J., Linzer D.I. and Wu B., Regulation of the human hsp70 promoter by p53. Science 259: 84-87, 1993.

34. van Oijen M.G. and Slootweg P.J., Gain-of-function mutations in the tumor suppressor gene p53. Clin. Cancer Res. 6: 2138-2145, 2000.

35. Dittmer D., Pati S., Zambetti G., Chu S., Teresky A.K., Moore M., Finlay C. and Levine A.J., Gain of function mutations in p53. Nat. Genet. 4: 42-46, 1993. 